\title{
Near-Earth Asteroids on Archival Schmidt Plates
}

\author{
R. H. McNaught, D. I. Steel ${ }^{1}$, K. S. Russell \\ Anglo-Australian Observatory, Private Bag, Coonabarabran, NSW 2357, \\ Australia \\ G. V. Williams \\ Harvard-Smithsonian Center for Astrophysics, 60 Garden Street, \\ Cambridge, MA 02138, U.S.A.
}

\begin{abstract}
We describe our routine identification of images of asteroids and comets on plates and films in the U.K. Schmidt Telescope archive. The asteroids of most interest to us are those which approach the Earth, and whenever such an object is found (by anyone) we perform backintegrations in order to determine whether the object may have been recorded on any UKST plate taken since 1973 . In many cases the object is found ('precovered') and measured, allowing an accurate orbit to be determined soon after its discovery; other studies such as long-term dynamical investigations, or predictions of future close approaches to the Earth, are then possible. Similar programs using other wide-field plate archives are to be encouraged, since valuable scientific results may be derived.
\end{abstract}

\section{Introduction}

The plate archives produced by Schmidt telescopes form a plentiful source of material for improving our knowledge of the orbits of asteroids. Since the AngloAustralian Near-Earth Asteroid Survey (AANEAS) began in 1990 (Steel et al. 1994) we have been routinely searching for pre-discovery trails of interesting and unusual asteroids using the plate archive produced by the U.K. Schmidt Telescope (UKST). The plates and, latterly, films (Parker et al. 1994) exposed with the UKST are stored partly at the telescope site, although the main archive is at the U.K. Schmidt Telescope Unit (UKSTU) at the Royal Observatory, Edinburgh (ROE); from its inauguration in 1973 through to mid-1988 the UKST was an outstation of the ROE, since when it has been operated as part of the Anglo-Australian Observatory.

By identifying and measuring asteroid/comet trails on archival plates the precision of the orbit determined for the object in question can be dramatically improved since a much extended time-base may be achieved. This allows future observations with narrow-field instruments since the ephemeris is then sufficiently well determined for easy acquisition telescopically. In addition, the

\footnotetext{
${ }^{1}$ Also with the Department of Physics and Mathematical Physics, University of Adelaide, Adelaide, SA 5005, Australia.
} 
orbital evolution and possibility of close approaches to the Earth can be investigated with much greater accuracy once a good orbit is available. Searches of the plate archive for possible pre-discovery detections are therefore highly desirable, and thus Schmidt (or other wide-field telescope) plate and film archives are important resources in studies of asteroids and comets.

\section{2. (2060) Chiron and (5145) Pholus}

One of the most celebrated cases involving an archival search was in the case of outer solar system object $(2060)$ Chiron $=1977$ UB. Images of this object have been found from as far back as 1895 (Kowal et al. 1979). Astrometric measurements of these images made the determination of an accurate orbit possible, and thus numerical integrations to investigate its past and future orbit (e.g. Hahn \& Bailey 1990).

Recently a similar object, (5145) Pholus = $1992 \mathrm{AD}$, was found by the University of Arizona Spacewatch team. The earliest images of Pholus were measured from UKST plates taken in 1977 and 1982. These images were identified following the 'precovery' (or pre-discovery recovery) of the object by E.M. and C.S. Shoemaker on films taken with the $0.46 \mathrm{~m}$ Palomar Schmidt, and by J. Mueller on plates taken with the $1.2 \mathrm{~m}$ Palomar Schmidt. With astrometric positions stretching over $\sim 15$ years being available soon after its identification, this asteroid very quickly had its orbit determined (and hence was numbered and named), making orbital evolution studies possible (Asher \& Steel 1993).

\section{Near-Earth Asteroids}

The main aim of the AANEAS program is the identification of near-Earth asteroids (NEAs), and the determination of their orbits. NEAs are usually discovered during a limited period of observability. When an asteroid of interest has a semireliable orbit determined, the plate log of the UKST is searched for candidate plates which may contain an image, either in that apparition, or a much earlier one. Orbital elements back-integrated to 1973 (i.e. when UKST operations began) are provided for this purpose by G.V.Williams. In some cases, no candidate plates are found. This often occurs when an NEA has periods of observability only at northern declinations, never coming within the grasp of the UKST. Additionally, since smaller/fainter asteroids are more difficult to detect and are generally only recorded at low geocentric distances their previous detection is unlikely.

Of the NEAs, the most successful searches generally are for large Amor asteroids. The larger objects can be recorded at greater geocentric distances and Amors, which are in orbits which approach but do not cross the Earth's orbit (perihelion distance $1.0167<q<1.3 \mathrm{AU}$ ), often have long phases of observability around opposition. For example, the large Amor asteroid (4954)1990 SQ was identified and measured on five UKST archival plates from 1975, allowing it to be numbered. Perhaps more unusually, the large Apollo (4953) $1990 \mathrm{MU}$ was found on six UKST plates back to 1974, again allowing numbering despite its having been observed on only three nights in 1990 (Apollos cross the Earth's orbit and have periods longer than one year). Only a handful of Aten-type asteroids (Earth-crossing but period less than one year) are known, making 
Table 1. NEAs on archived UKST plates. The numbers in brackets indicate how many pre-discovery archival plates held images of the asteroids.

\begin{tabular}{|c|c|c|c|c|}
\hline \multicolumn{5}{|c|}{ Apollos (Earth-crossing, $a \geq 1 \mathrm{AU}, q \leq 1.0167 \mathrm{AU}$ ) } \\
\hline $\begin{array}{rr}\text { (4015) } 1979 \mathrm{VA} & {[1]} \\
1990 \mathrm{BG} & {[1]} \\
1992 \mathrm{JB} & {[2]}\end{array}$ & $\begin{array}{l}\text { (4953) } 1990 \mathrm{MU}[6] \\
1990 \mathrm{HA}^{[}[1] \\
1992 \mathrm{UY}_{4}[2]\end{array}$ & 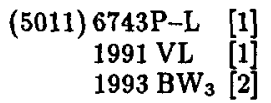 & $\begin{array}{l}1987 \mathrm{OA} \\
1992 \mathrm{CC}_{1} \\
1993 \mathrm{EA}\end{array}$ & $\begin{array}{l}{[1]} \\
{[1]} \\
{[4]}\end{array}$ \\
\hline \multicolumn{5}{|c|}{ Atens (Earth-crossing, $a<1 \mathrm{AU}, Q \geq 0.9833 \mathrm{AU}$ ) } \\
\hline \multicolumn{5}{|c|}{ Amors $(1.0167<q \leq 1.3 \mathrm{AU})$} \\
\hline $\begin{array}{c}(4660) 1982 \mathrm{DB} \\
(4954) 1990 \mathrm{SQ} \\
(5407) 1992 \mathrm{AX} \\
1988 \mathrm{VN}_{4}[1] \\
1991 \mathrm{OA}^{3}\end{array}$ & $\begin{array}{rr}(4775) 1927 \mathrm{TC} & {[1]} \\
(4957) 1990 \mathrm{XJ} & {[4]} \\
(5587) 1990 \mathrm{SB} & {[2]} \\
1990 \mathrm{TR} & {[2]} \\
1992 \mathrm{AA} & {[1]}\end{array}$ & $\begin{aligned}(4947) 1988 \mathrm{TJ}_{1} & {[1] } \\
(5332) 1990 \mathrm{DA} & {[2] } \\
(5626) 1991 \mathrm{FE} & {[2] } \\
1990 \mathrm{WA} & {[2] } \\
1992 \mathrm{JE} & {[2] }\end{aligned}$ & $\begin{array}{c}(5653) 1992 \mathrm{WD}_{5} \\
1991 \mathrm{CQ} \\
1992 \mathrm{TC}\end{array}$ & $\begin{array}{l}{[4]} \\
{[1]} \\
1]\end{array}$ \\
\hline
\end{tabular}

(3753) 1986 TO, an Aten discovered at the UKST, especially noteworthy; it has also been identified on six archival plates (as a consequence of having a period of almost exactly one year). An account of the peculiar discovery circumstances of Amor asteroid $1977 \mathrm{QQ}_{5}$ has been given by Steel et al. (1994). These are large objects which are brightest in the southern skies and observable regularly.

A full list of the NEAs identified to date on UKST archive plates is given in Table 1 . We estimate that there are likely $>250$ NEA trails yet to be found on the $\sim 16,000$ plates in the archive.

\section{Main-Belt Asteroids}

Main-belt asteroids are typically represented on six to ten old plates. It is occasionally the case that a new discovery, with no identifications in the files of previously observed asteroids, can be sufficiently well observed from measures on archival plates to allow numbering during the discovery apparition. Examination of archival material can also produce discoveries, as was the case with $1988 \mathrm{BM}_{5}$ which was identified on three plates taken in that year. It was then found on six additional archival plates dating back to 1980 leading to its being numbered and named (4128) UKSTU, in recognition of the discovery telescope and the plate archive produced by that instrument.

\section{Other Programs}

Similar work to that described above has been done by $\mathrm{R}$. West and colleagues using the European Southern Observatory, Palomar and UKST plate archives, particularly in identifying the handful of 'lost' numbered asteroids; McNaught found a UKST image which confirmed the recovery of (878) Mildred by Williams (IAU Circ. 5275), making (719) Albert the only lost numbered asteroid. In addition to their discovery programs, the Shoemaker and Helin teams (see Steel 
et al. 1994) have a large archive of films taken with the $0.46 \mathrm{~m}$ Schmidt at Palomar, and they produce a number of identifications from these.

E. Bowell and colleagues at the Lowell Observatory have made, and continue to make, many identifications of NEAs and other unusual asteroids on the first Palomar survey and other materials, including their own extensive plate archive. Their most unusual success to date was in finding images of the Apollo asteroid 1979 VA on plates taken in 1949 during the first Palomar Sky Survey with the $1.2 \mathrm{~m}$ Schmidt. 1979 VA had been discovered in that year by E.F. Helin, but the 1949 images seemed cometary. It was quickly realised (Marsden 1992) that the 1949 images had been reported at the time as comet 1949 III (Periodic) Comet Wilson-Harrington; it is now known as (4015) Wilson-Harrington. This is the first clearly-demonstrated example of a comet turning into an asteroid, the cometary activity only being visible on the discovery plates from 1949, no activity being evident in 1979 (or since).

\section{Conclusions}

There is a wealth of useful information in Schmidt plate/film archives pertaining to earlier detections of asteroids and comets. Astrometric measurements of such images can lead to the rapid determination of accurate orbits for newlyrecognized objects. Inspection of the images may lead to discoveries of physical importance (e.g. cometary activity being identified in objects that now appear asteroidal). The involvement of all astronomers with access to wide-field plate archives is to be encouraged, with easily-performed back-integrations leading to the flagging of plates on which images might be found, and inspection of those plates rendering important astrometric positions which lead to valuable improvements in our knowledge of the orbits of the objects involved.

\section{Acknowledgments}

This work was financially supported by the Australian Research Council and the Department of Employment, Education and Training. Facilities were generously made available by the Anglo-Australian Observatory, and the Royal Observatory, Edinburgh. We would also like to acknowledge the contribution of the many UKST observers who, over the past two decades, have exposed the plates which have been used in this work, and the staff at the ROE plate library who have assisted in making these materials accessible.

\section{References}

Asher D. J. \& Steel D. I., 1993, MNRAS, 263, 179

Hahn G. \& Bailey M. E., 1990, Nature, 348, 132

Kowal C. T., Liller W. \& Marsden B. G., 1979, in Dynamics of the Solar System, R.L. Duncombe, ed., (Reidel, Dordrecht)

Marsden B. G., 1992, IAU Circ. 5585

Parker Q. A., Phillipps S. \& Morgan D. H., 1994, these proceedings

Steel D. I., McNaught R. H., Garradd G. J., Russell K. S. \& Asher D. J., 1994, these proceedings 